\title{
Seed Mediated Synthesis of Colloidal Halide Perovskite
}

\author{
Nanoplatelets \\ C. Meric Guvenc, ${ }^{\mathrm{a}}$ and Sinan Balci ${ }^{\mathrm{b}^{*}}$

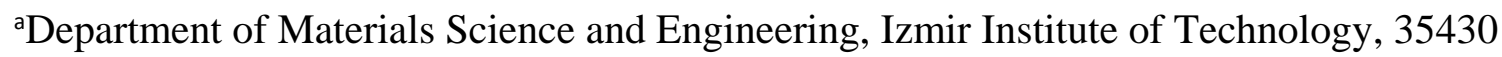 \\ Izmir, Turkey \\ ${ }^{\mathrm{b}}$ Department of Photonics, Izmir Institute of Technology, 35430 Izmir, Turkey \\ *E-mail: sinanbalci@iyte.edu.tr
}

\begin{abstract}
Two-dimensional lead halide perovskite nanoplatelets (2D LHP NPLs) have been emerging as one of the most promising semiconductor nanomaterials due to their narrow absorption and emission line widths, tunable bandgaps, high exciton binding energies, high defect tolerance as well as highly localized energy states. Colloidal synthesis of 2D LHP NPLs is generally performed using hot-injection or ligand assisted precipitation techniques (LARP). In the LARP method, perovskites are synthesized in polar solvents, which decrease the stability of the 2D LHP NPLs due to their weakly bonded nature. In fact, the presence of residual polar solvent in the LHP NPL colloid can cause deterioration of thickness uniformity, degradation of NPLs to parent precursors, and undesired phase transformations. Herein, for the first time, we report facile seedmediated synthesis route of monolayer, 2-monolayers, and thicker lead halide perovskite nanoplatelets without using A site cation halide salt (AX; A = Cesium, methylammonium, formamidinium and, $\mathrm{X}=\mathrm{Cl}, \mathrm{Br}, \mathrm{I})$ and long chain alkylammonium halide salts ( $\mathrm{LX}$; $\mathrm{L}=$ oleylammonium, octylammonium, butylammonium and, $\mathrm{X}=\mathrm{Cl}, \mathrm{Br}$, I). The seed solution has been synthesized by reacting lead (II) halide salt and coordinating ligands (oleylamine or octylamine and oleic acid) in nonpolar high boiling solvent (1-octadecene). The seed
\end{abstract}


mediated synthesis has been carried out in hexane by reacting seed solution with A-site cation precursors (Cs-oleate, FA-oleate, or diluted MA solution in hexane) under ambient conditions. More importantly, the seed mediated growth of NPLs has been tracked for the first time by performing in-situ optical measurements. Furthermore, the optical properties and morphologies of the seeds have been extensively studied. We find that our facile synthesis route provides highly stable, monodisperse NPLs with narrow absorption, and photoluminescence line widths (68-201 meV), and high PLQY (37.6-1.66\% for 2ML NPLs). Furthermore, anion exchange reactions have been performed by mixing presynthesized LHP NPLs with counter halide seeds. The optical properties of NPLs have been affectively tuned by postsynthetic chemical reactions without changing the thickness of the NPLs. We anticipate that our new synthetic route provides further understanding of growth dynamics of LHP NPLs.

\section{INTRODUCTION}

Lead halide perovskite nanoplatelets have drawn a tremendous amount of attention due to their unique optical properties. Their narrow absorption and emission line widths, tunable bandgaps, high exciton binding energies, easy of synthesis, high defect tolerance, and highly localized energy levels make them a great candidate for the state of the art electronic, optoelectronic, and photonic technologies. ${ }^{1-7}$ Generally, colloidal 2D LHP NPLs have been synthesized using LARP or hot-injection techniques. ${ }^{1,5,8-12}$ In the LARP technique, LX, lead halide $\left(\mathrm{PbX}_{2}\right)$, and $\mathrm{AX}$ salts are dissolved in a polar solvent such as dimethylformamide (DMF) or dimethyl sulfoxide (DMSO) and subsequently, proper amounts of these solutions are directly injected into nonsolvents such as toluene or hexane..$^{5,8,13-15}$ Furthermore, synthesis of organolead halide perovskite NCs in nonsolvent media has been demonstrated by using ligandmediated transport methods. However, ligand mediated transport method only supports 
synthesis of LHP NCs, and therefore, controlling quantum size effects at monolayer thickness has not yet been achieved by using this technique. ${ }^{16}$ Vybornyi et al. reported polar solvent free synthesis of MAPbBr3 NPLs and NCs by using ionic metathesis reaction. ${ }^{17}$ In addition, Bekenstein et al. have recently reported synthesis of $\mathrm{CsPbBr} 3$ NPLs from 1-to-5-unit cells by using the modified hot-injection process and hence they have indeed tuned the optical properties of NPLs in the entire visible region by using halide exchange reactions. ${ }^{10}$ On the other hand, seed-mediated synthesis of strongly quantum confined $\mathrm{MAPbI}_{3}$ nanocrystals has been recently demonstrated. ${ }^{18}$ This procedure requires synthesis of lead oleate and preparation of halide precursor by dissolving tetrabutylammonium iodide in oleylamine and 1-octadecene mixture at relatively high temperatures and long times. Furthermore, synthesized lead oleate and halide precursors were reacted at $200{ }^{\circ} \mathrm{C}$ for the synthesis of $\mathrm{PbI}_{2} \mathrm{NC}$ seeds and then, $\mathrm{PbI}_{2} \mathrm{NC}$ seeds were reacted with MAI salt in toluene and chloroform mixture for the synthesis of $\mathrm{MAPbI}_{3}$ nanocrystals. ${ }^{18}$ In another recent study, Huang et al. have demonstrated synthesis of LHP thick NPLs and nanocubes in a nonpolar solvent by carrying out spontaneous crystallization. ${ }^{9}$ Also, they claimed that the mechanism of the synthesis was a seed mediated growth. However, they were not able to observe the optical properties of the synthesized seeds and growing steps of NCs due to the extremely fast reaction rate. ${ }^{9}$ Udayabhaskararao et al. claimed that $\mathrm{Pb}^{\circ}$ metallic seeds were formed in the early stage of the hot injection method and, the $\mathrm{Pb}^{\mathrm{o}}$ seeds provide nucleation sites for the perovskite layers. ${ }^{19}$ Nevertheless, in the previous studies, the evolution of LHP NCs from seeds was not demonstrated by performing ultraviolet-visible absorption or photoluminescence measurements. Here, for the first time, we show seed-mediated synthesis of very thin LHP NPLs with widely used ligands and precursors in a hot injection method. ${ }^{17,20 \text {, }}$ ${ }^{21}$ The uniqueness of this method allows us to synthesize monolayer, two monolayers, and thicker nanoplatelets without using LX or AX salts and polar solvents. Notably, LHPs have weak structural bonds and also their bonds with the ligand shells are very weak and highly 
dynamic, making them very sensitive to polar solvents. In fact, performing synthesis of LHP NPLs in polar solvents can decrease the stability of the NPLs due to their weakly bonded nature. ${ }^{9,22}$ The deterioration of thickness uniformity, ${ }^{8}$ dissociation to precursor salts, ${ }^{23-25}$ and transformation to wide and indirect band gap phases ${ }^{26,27}$ are main problems for 2D LHP NPLs and all LHPs. These problems can be primarily triggered and promoted by the presence of residual polar solvents in the colloid. Importantly, our facile synthesis route provides highly stable, monodisperse NPLs with narrow absorption, photoluminescence line widths, and high PLQY. Additionally, synthesized seeds can be directly used in halide exchange reactions by preserving the uniform thickness of the nanoplatelets. The halide exchange reaction with the seeds indeed provides easy tuning of the LHP NPLs band gap. Finally, the seed mediated growth of NPLs was monitored continuously for the first time by in situ ultraviolet-visible absorption measurements.

\section{RESULTS AND DISCUSSION}

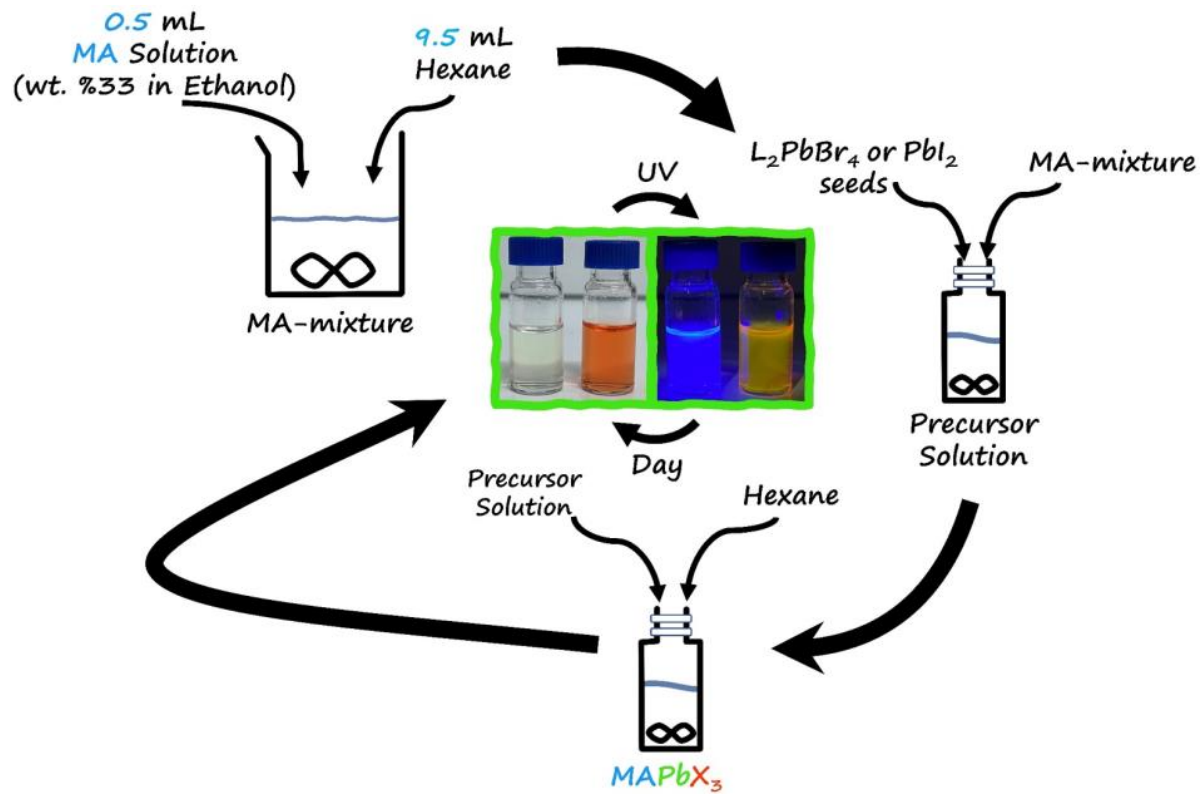

Figure 1. Schematic representation of facile synthesis route of $2 \mathrm{ML}$ MAPbX 3 NLPs. Briefly, proper amount of prepared MA-mixture was mixed with seeds. The obtained seed solution was mixed with hexane for the synthesis of $2 \mathrm{ML}$ MAPbX $\mathrm{X}_{3}$ NPs. The synthesis 
of $\mathrm{MAPbBr}_{3}$ NPLs takes a longer time than $\mathrm{MAPbI}_{3} \mathrm{NPLs}_{\text {due }}$ to the stronger $\mathrm{Pb}-\mathrm{Br}$ bonds. The same synthesis route was used for all A site cations (Cs, MA, and FA) used in this work. Cs-oleate and FA-oleate were used as A-site cation precursor for the synthesis of CsPbX3, and FAPbX3 NPLs, respectively. Synthesis of CsPbBr 3 and FAPbBr 3 NPLs took a longer time than iodide counterparts, similar to the MAPbBr 3 NPLs.

Schematic representation of the seed mediated synthesis of 2ML and thicker LHP NPLs is demonstrated for $\mathrm{MAPbX}_{3} 2 \mathrm{ML}$ NPLs in Figure 1. The same synthesis route can be also used for the synthesis of $\mathrm{CsPbX}_{3}$, and $\mathrm{FAPbX}_{3}$ NPLs. In fact, only A site cation precursor was changed to Cs-oleate and FA-oleate for the synthesis of $\mathrm{CsPbX}_{3}$ and $\mathrm{FAPbX}_{3}$ NPLs, respectively. It should be emphasized that, in the described method, only minuscule amount of residual polar solvent exists in $\mathrm{MAPbX}_{3}$ NPLs. Nevertheless, residual polar solvent is at least ten times less than the LARP method. ${ }^{8}$ Diluted MA solution (33 wt. \% in absolute ethanol) in hexane was used for ionic metathesis reaction. Note that, in the metathesis reaction, the proton required for the formation of $\mathrm{CH}_{3} \mathrm{NH}_{3}{ }^{+}$comes from the oleic acid. ${ }^{17,}{ }^{20}$ In the seed-mediated synthesis, MA was protonated by the available oleic acid molecules in the seed solutions. Around $30 \mu \mathrm{L}$ of diluted MA solution, which contains $1.92 \mu \mathrm{L}$ of ethanol, was used in the synthesis of thick $\mathrm{MAPbI}_{3} \mathrm{NPLs}$. In order to synthesize $2 \mathrm{ML} \mathrm{MAPbI}_{3}$ and $\mathrm{MAPbBr}_{3} \mathrm{NPLs}$, the MA solution contains $0.16 \mu \mathrm{L}$ and $0.8 \mu \mathrm{L}$ ethanol, respectively. Indeed, the explained procedure enables facile synthesis of 2ML and thick NPLs. Also, to synthesize Cs-oleate and FA-oleate precursors, $\mathrm{CsCO}_{3}$ and formamidine acetate salts were reacted with OA. Subsequently, the synthesized Cs-oleate or FA-oleate precursors were mixed with the seed solution, and then quickly injected into hexane for the synthesis of $\mathrm{CsPbX}_{3}$ or $\mathrm{FAPbX}_{3} \mathrm{NPLs}$. 

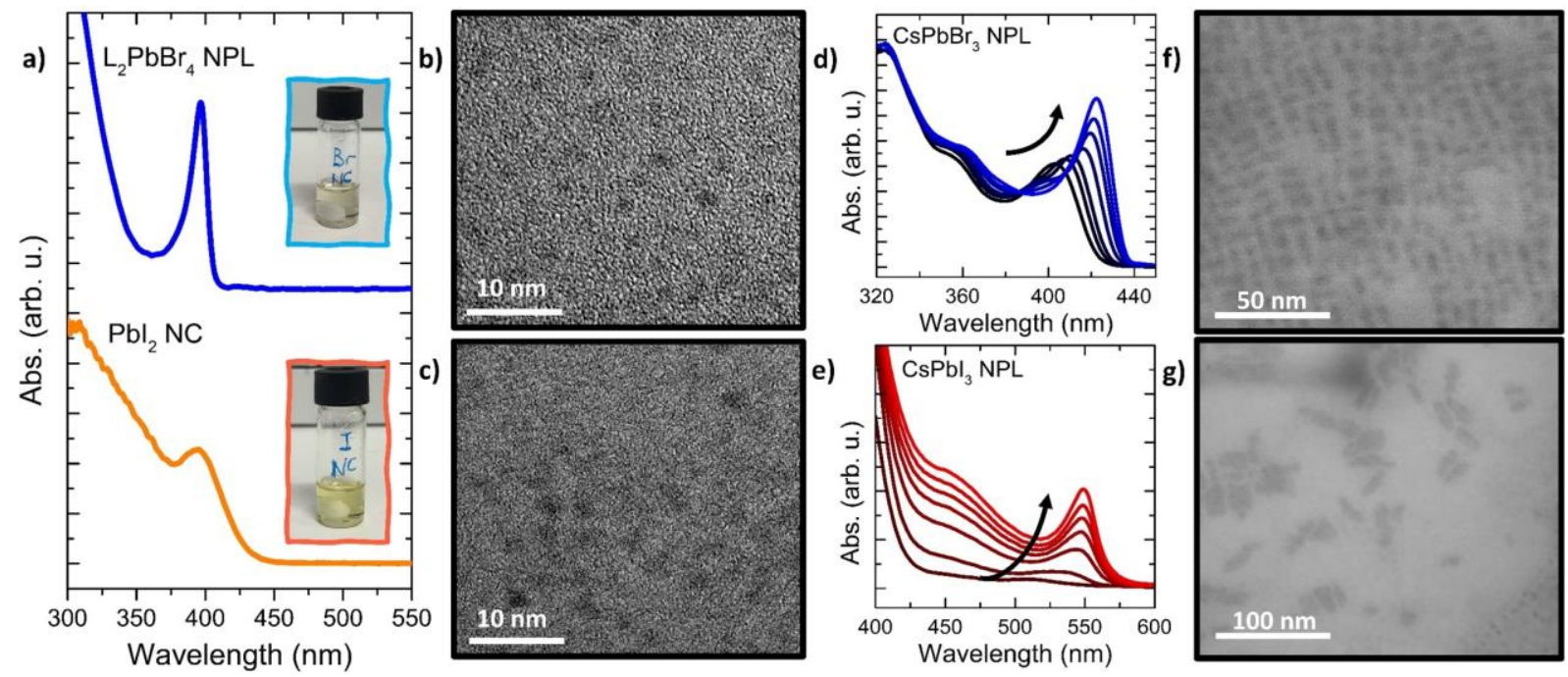

Figure 2. Seed mediated synthesis of $2 \mathrm{ML} \mathrm{CsPbBr} 3$ and $\mathrm{CsPbI}_{3} \mathrm{NPLs}_{3}$ (a) Absorption spectra of $\mathrm{L}_{2} \mathrm{PbBr}_{4}$ and $\mathrm{PbI}_{2}$ seeds. The NC seeds were synthesized from commonly used hot injection precursors. $\mathrm{PbX}_{2}$ salt was dissolved in 1-octadecene by using oleic acid, and oleylamine at $150{ }^{\circ} \mathrm{C}$ and then the reaction was quenched by immersing in an ice-water bath. (b,c) TEM images of $\mathrm{L}_{2} \mathrm{PbBr}_{4}$ NPLs and $\mathrm{PbI}_{2} \mathrm{NCs}$, respectively. In-situ absorption spectra of (d) $2 \mathrm{ML} \mathrm{CsPbBr} 3$ and (e) $2 \mathrm{ML} \mathrm{CsPbI}_{3}$ NPLs in hexane during the seedmediated formation of NPLs; each spectrum is taken after $1 \mathrm{~min}$. STEM images of (f) 2 ML CsPbBr 3 NPLs, and (g) 2 ML CsPbI 3 NPLs.

Figure 2a shows absorption spectra of $\mathrm{L}_{2} \mathrm{PbBr}_{4}$ (L=oleylammonium) NPLs and $\mathrm{PbI}_{2}$ seeds and their corresponding TEM images were shown in Figures $2 b$ and $2 c$. The synthesized NCs are nearly in uniform circular shapes and their diameters are around 2-3 nm. Furthermore, the $\mathrm{L}_{2} \mathrm{PbBr}_{4} \mathrm{NPLs}$ and $\mathrm{PbI}_{2}$ NCs (see Supporting Information Figure S1) scatter incident red laser light, and hence observation of Tyndall effect suggests the existence of the seed nanocrystal colloid. It should be noted that the synthesized $\mathrm{PbI}_{2} \mathrm{NC}$ seeds demonstrated in this work show similar optical and morphological characteristic with the previously synthesized NCs by Hassan et al. ${ }^{18}$ In fact, the synthesized $\mathrm{L}_{2} \mathrm{PbBr}_{4} \mathrm{NC}$ seeds have an excitonic absorption 
peak around $397 \mathrm{~nm}$, which is nearly the same absorption position to the previously demonstrated $\mathrm{L}_{2} \mathrm{PbBr}_{4}$ NPLs excitonic absorption peak by Weidman et al. ${ }^{8}$ It is noteworthy that $\mathrm{L}_{2} \mathrm{PbBr}_{4} \mathrm{NPL}$ seeds were synthesized by using the same procedure as $\mathrm{PbI}_{2} \mathrm{NC}$ seeds. The $\mathrm{PbI}_{2}$ crystals, being a layered structure, consists of strongly bonded I-Pb-I layers where weak van der Waals forces are present in interlayers. ${ }^{28}$ Indeed, intercalation of A site cations to the layered $\mathrm{PbI}_{2}$ structures produces perovskite structure. ${ }^{29}$ In the seed mediated synthesis of $\mathrm{APbBr}_{3} \mathrm{LHP}$ NLPs, the $\mathrm{L}_{2} \mathrm{PbBr}_{4}$ NPLs were used as seeds, which have only single layer $\mathrm{PbBr}_{6}$ octahedrons. ${ }^{5}$, ${ }^{8,30}$ Adding A site cations to the seed solution (layered structure) produces $\mathrm{APbBr}_{3}$ type $3 \mathrm{D}$ perovskite structure, and therefore, we obtain 2ML or thick NPLs in the seed mediated synthesis. The growth of $2 \mathrm{ML} \mathrm{CsPbBr} 3$ and $\mathrm{CsPbI}_{3}$ NPLs were followed by in situ ultravioletvisible absorption measurements. Figures $2 \mathrm{~d}$ and $2 \mathrm{e}$ show variation of absorption spectra of the colloid during the growth of the nanoplates. In the first stage, small LHP NCs, having a very broad excitonic absorption peak, are formed. In the later stage of the nanocrystal growth, the excitonic absorption peaks shift to longer wavelengths, and concurrently the linewidth of the excitonic absorption peak is narrowed. In the final stage of the growth, $2 \mathrm{ML} \mathrm{CsPbBr}_{3}$ and $\mathrm{CsPbI}_{3} \mathrm{NPLs}$ are formed and excitonic absorption peaks are observed at 429 and $556 \mathrm{~nm}$, respectively. The STEM images in Figures $2 \mathrm{f}$ and $2 \mathrm{~g}$ reveal that the nanoparticles are of platelet (2ML NPLs) morphology. In fact, a close inspection of the STEM images indicates that the $\mathrm{CsPbBr}_{3}$ NPLs have very small lateral dimensions, which are close to $10 \mathrm{~nm} \times 3 \mathrm{~nm}$. On the other hand, the $\mathrm{CsPbI}_{3}$ NPLs have at least two times larger lateral dimension than the $\mathrm{CsPbr}_{3}$ NPLs. This may be due to the fact that the weak chemical bonds between Pb-I atoms are more easily broken and reformed than the chemical bonds between $\mathrm{Pb}-\mathrm{Br}$ atoms. ${ }^{31,32}$ Therefore, the size of the $\mathrm{CsPbI}_{3}$ NPLs are larger than the $\mathrm{CsPbBr}_{3}$ NPLs after terminating the growth reaction at the same time period. It should be emphasized here that the synthesized NPLs in this work have very small lateral dimensions than the previously reported nanoplatelets, ${ }^{8,13}$ which might 
be due to the growth of NPLs from individual nanocrystal seeds in this study. We should note here that in order to fully understand the growth mechanism of nanoplatelets from small nanocrystals further studies are urgently required.

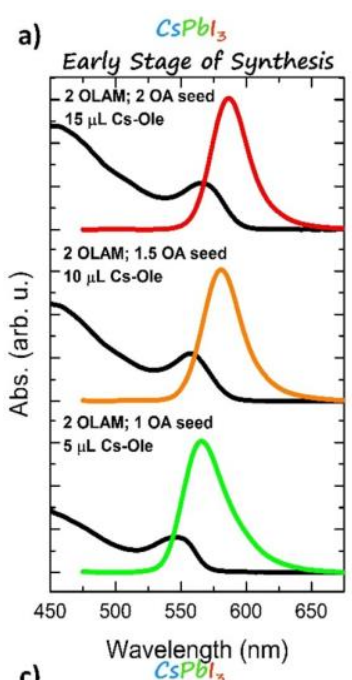

c)
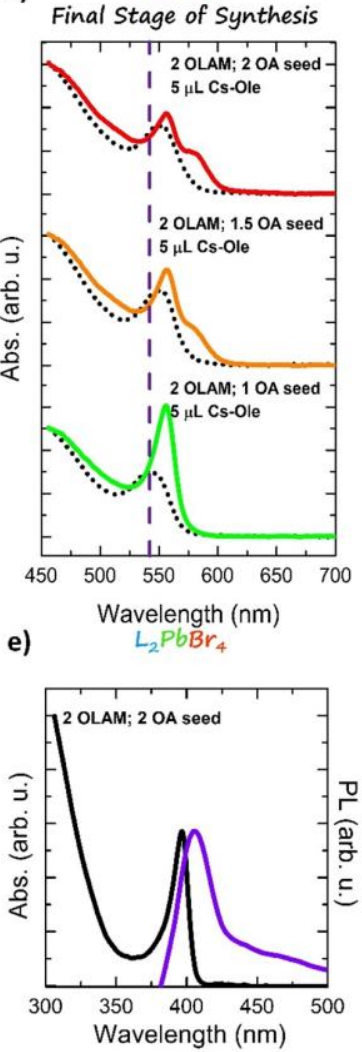

b) $\mathrm{CsPbl}_{3}$

Final Stage of Synthesis

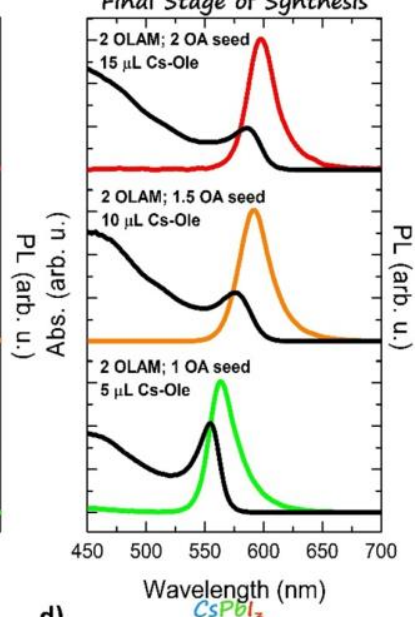

d)

Final Stage of Synthesis

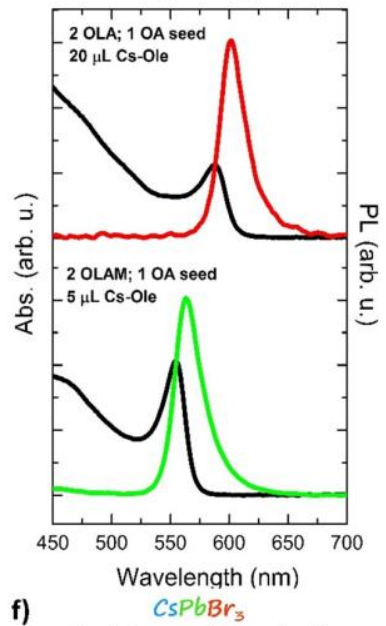

Final Stage of Synthesis

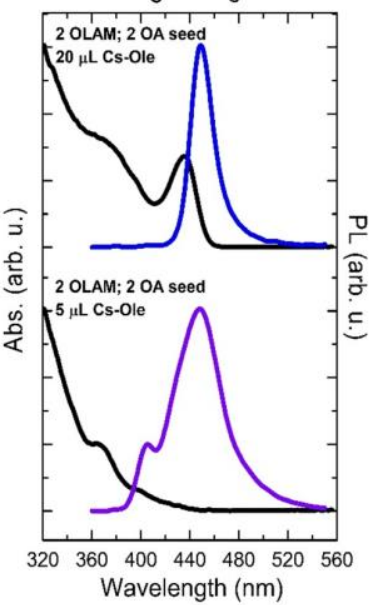


Figure 3. Seed mediated growth of perovskite nanoplatelets. (a,b) The early and final stages of the seed-mediated synthesis. The variation of the ligand composition of the seed solution and Cs-oleate amount allow us to tune thickness of NPLs. The thickness of nanoplatelets tends to increase with a decrease in OLAM/OA ligand volume ratio in the seed solutions. By decreasing OLAM/OA volume ratio used in NC seeds from 2:1 to 2:1.5 and then to 2:2, the amount of Cs-oleate required for the synthesis of monodisperse NPLs increases from $5 \mu \mathrm{L}$ to $10 \mu \mathrm{L}$ and then to $15 \mu \mathrm{L}$. (c) The Cs-oleate solution amount is fixed to $5 \mu$ l while the seeds have varying ligand volume ratios of OLAM/OA2: $x(x=1-2)$. The increasing $\mathrm{OA}$ concentration in seed solution causes heterogeneous thickness dispersion of NPLs when fixed amount $(5 \mu \mathrm{l})$ Cs-oleate is used. The thickness of the nanoplatelets strongly depends on the OLAM/OA volume ratio of the seed solution used in the synthesis. Besides, to synthesize monodisperse NPLs, the amount of Cs-oleate solution added to the seed solution is very crucial. (d) Monodisperse thicker NPLs were obtained by increasing Cs-oleate from $5 \mu$ lo $20 \mu$ l for seeds with an OLAM/OA volume ratio of 2:1. (e) Absorption and PL spectra of $\mathrm{L}_{2} \mathrm{PbBr}_{4}$ NPLs, which have been used as seeds in the synthesis of nanoplatelets. (f) Absorption and PL spectra of $\mathrm{CsPbBr}_{3}$ NPLs synthesized from seeds that have OLAM/OA volume ratio of $2: 2$ by using $20 \mu \mathrm{L}$ and $5 \mu$ l Cs-oleate.

We now turn our attention to understand the effects of the ligand volume ratio and Csoleate amount on the synthesis of monodisperse LHP NPLs. In order to reach this goal, seeds with varying ligand volume ratios (OLAM/OA $=2: 1,2: 1.5,2: 2)$ were prepared and subsequently, the seeds were reacted with various Cs-oleate amounts, see Figure 3a. For example, the seeds having OLAM/OA volume ratio of 2:1 were reacted with $5 \mu \mathrm{L}$ of Cs-oleate and when this ligand volume ratio was decreased to 1, the Cs-oleate amount was increased to $15 \mu \mathrm{L}$. The absorption measurements reveal that, at the beginning of the synthesis, the 
absorption peaks of NCs shifted to longer wavelengths with a decrease in OLAM/OA volume ratio. Therefore, the red shift observed in the absorption spectra implies that increasing OA amount results in formation of slightly larger NCs. As the reaction proceeds, the linewidth of the excitonic absorption peaks decreases with a slight red shift in the absorption peak position, see Figure $3 \mathrm{~b}$ for the absorption and emission peaks. On the other hand, similar experiments were performed by keeping the Cs-oleate amount constant, $5 \mu 1$, in the reactions for all the ligand volume ratios (Figure 3c). However, the absorption spectra in Figure $3 \mathrm{c}$ indicate that the thickness of the NPLs is not homogeneous. This also implies that increasing OA amount in the seed NCs causes an increase in NPL thickness and hence only specific amounts of OA and the A site cation ratios generate homogeneous NPL thickness. In order to synthesize $2 \mathrm{ML} \mathrm{APbX} 3$ NPLs, low concentration of A site cation and high alkylamine concentration are required. For example, Cho et al. observed the similar trend in the synthesis of $\mathrm{MAPbBr} 3 \mathrm{NPLs}^{13}{ }^{13}$ In addition, 2ML and thicker NPLs were successfully synthesized by using the seed mediated synthesis where Cs-oleate amount was increased from $5 \mu \mathrm{L}$ to $20 \mu \mathrm{L}$ for a constant OLAM/OA volume ratio of 2, see Figure 3d. However, we were not able to synthesize $2 \mathrm{ML} \mathrm{APbI}_{3} \mathrm{NPLs}$ by using lower ligand volume ratios than 2:1 in the seed mediated synthesis. Furthermore, the absorbance and PL spectra of $\mathrm{L}_{2} \mathrm{PbBr}_{4}$ NPLs were shown in Figure 3e. It should be emphasized that in previous studies, $\mathrm{L}_{2} \mathrm{PbBr}_{4}$ NPLs were reported to have very low PLQY and instability at ambient conditions. ${ }^{8}$ The $\mathrm{L}_{2} \mathrm{PbBr}_{4}$ NPL seeds having OLAM/OA volume ratio of 2:1 rapidly aggregates within hours even in the crude solution and hence the $\mathrm{L}_{2} \mathrm{PbBr}_{4}$ NPL seeds with a ligand volume ratio of 2:1 should be used immediately. However, the synthesized $2 \mathrm{ML}$ and thick NPLs are very stable. In particular, all inorganic 2ML and thick LHP NPLs have very high stability. The higher stability of NPLs might be due to the combined use of oleylamine and oleic acid to stabilize NLPs in our seed mediated synthesis method. In fact, the combination of oleylamine and oleic acid forms oleylammonium oleate, which bounds relatively tightly to 
NPL surface in a similar to the oleyammonium halides. ${ }^{33}$ Therefore, our method yields more stable NPLs than the previously synthesized NPLs by using the LARP technique. ${ }^{8}$ It is clear in Figure $3 \mathrm{f}$ that $\mathrm{CsPbBr} 3$ NPLs could not be synthesized at room temperature by using $5 \mu \mathrm{L}$ of Cs-oleate. The incompletion of growth of the $\mathrm{CsPbBr}_{3}$ NPLs was observed when $5 \mu 1$ of Csoleate is used in the seed mediated synthesis. In Figure 3f, the small shoulder observed at wavelengths around $400 \mathrm{~nm}$ is most likely originated from the $1 \mathrm{ML} \mathrm{L}_{2} \mathrm{PbBr}_{4}$ seeds. The wider peak covering the wavelength range from 380 to $520 \mathrm{~nm}$ also confirms the incomplete formation of $\mathrm{CsPbBr}_{3}$ NPLs. Besides, we have successfully achieved synthesis of thick $\mathrm{CsPbBr}_{3}$ NPLs by using $20 \mu \mathrm{L}$ of Cs-oleate during the seed mediated synthesis as shown in Figure 3f.
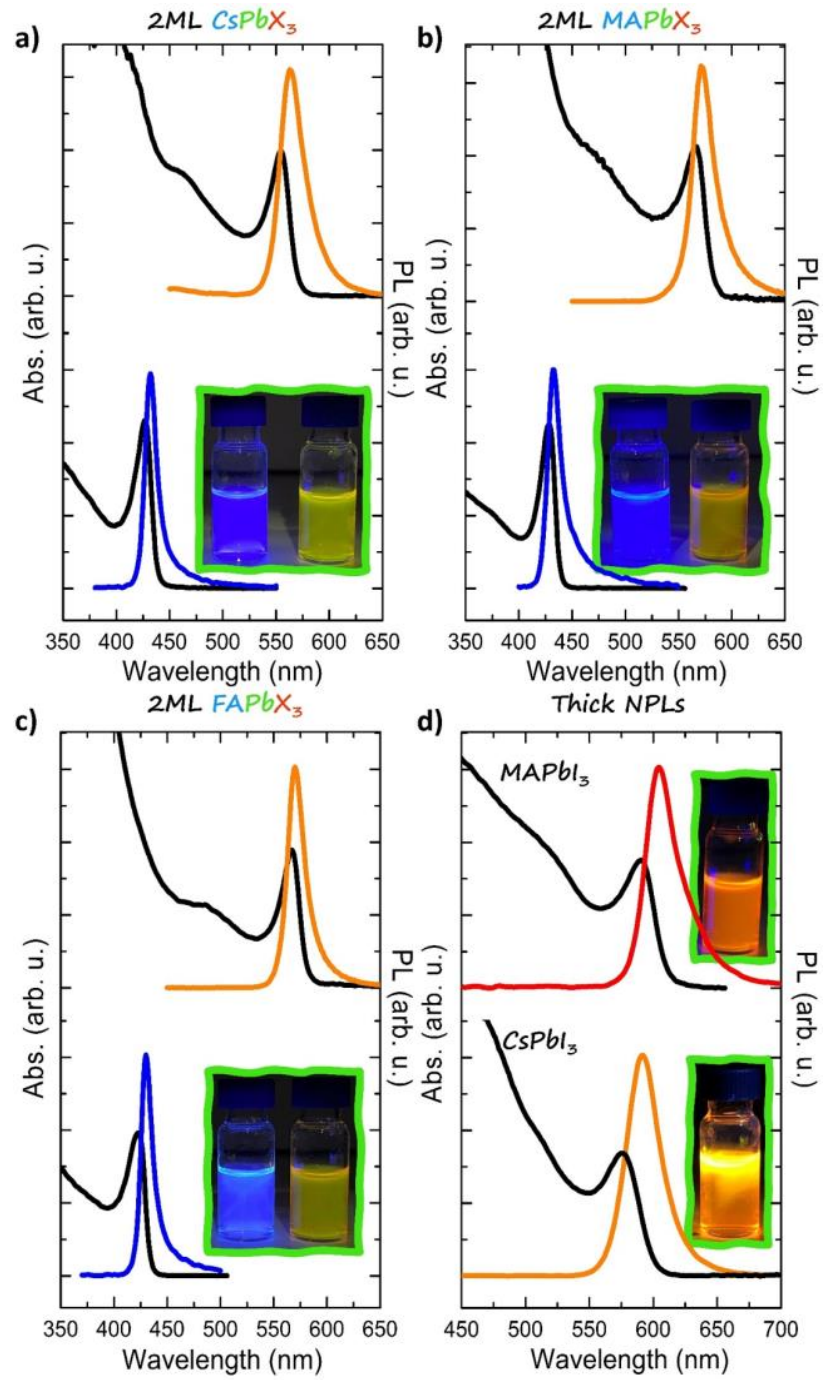
Figure 4. Seed mediated synthesis of 2ML and thick nanoplatelets. Absorption and PL spectra of (a) $2 \mathrm{ML} \mathrm{CsPbX}$, (b) $\mathrm{MAPbX}_{3}$, (c) $\mathrm{FAPbX}_{3}$, and (d) thick $\mathrm{CsPbI}_{3}$, and $\mathrm{MAPbI}_{3}$ NPLs synthesized by using the seed-mediated route. The inset in the graphs display the photos of the emitting nanoplatelets under $350 \mathrm{~nm}$ UV light illumination. In (a), (b), and (c), the photos of 2ML APbBr 3 NPLs and 2ML APbI 3 NPLs are on the left-hand side of the photos, and on the right-hand side of the photos, respectively. The inset in (d) shows the photographs corresponding to thick $\mathrm{MAPbI}_{3}$ in the upper side and $\mathrm{CsPbI}_{3} \mathrm{NPLs}_{\mathrm{s}}$ in the lower side of the graph.

To examine the effect of NPL thickness on the optical properties of the NPLs, 2ML and thick NPLs were synthesized using the seed mediated synthesis route. The absorption and PL spectra of the synthesized NPLs were all shown in Figure 4. In addition, the inset photographs show emission of colloidal LHP NPLs under $350 \mathrm{~nm}$ UV light illumination. Note that for all A site cations used in this work, seed mediated synthesis of $\mathrm{APbBr}_{3}$ NPLs takes a longer time then $\mathrm{APbI}_{3}$ NPLs. Further, organo-lead halide perovskite NPLs were synthesized much faster than all inorganic counterparts for both $\mathrm{APbBr}_{3}$ and $\mathrm{APbI}_{3} \mathrm{NPLs}$. The faster synthesis may indicate higher diffusion kinetics of organic cations $\left(\mathrm{MA}^{+}, \mathrm{FA}^{+}\right)$than inorganic cations $\left(\mathrm{Cs}^{+}\right)$. The growth of all inorganic LHP NPL can be accelerated by heating. Detailed procedures were explained in the methods section. The narrowest linewidths in PL emission spectra were obtained in the nanoplatelet colloids synthesized by using FA cation as shown in Figure 4c, which is also observed in the previous studies. ${ }^{8}$ Particularly, the seed mediated synthesis of LHP NPLs does not require any additional purification steps since the seed mediated synthesis provides homogenous NPL thickness. In addition, homogeneous thickness controlled NPLs can be obtained using Cs and MA cations in the synthesis of thick NPLs, see also Figure S2. However, we were not able to synthesize homogenous thick NPLs by using FA cation. Thus, 
increasing FA-oleate amount from $6 \mu \mathrm{L}$ to higher amounts results in synthesis of NPLs with heterogeneous thickness as shown in Figure S3. Additionally, our facile and simple method allows large-scale synthesis of perovskite NPLs as shown in Figure S4. We also examined the stability of perovskite NPLs, see Figure S5. A slight redshift was observed in the absorption and PL spectra of the NPLs with time. However, the seed synthesized NPLs were preserved thickness uniformity as shown in Figure S5.

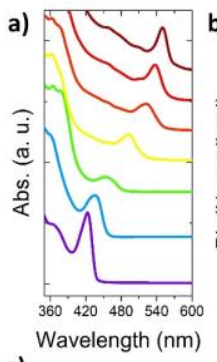

c)
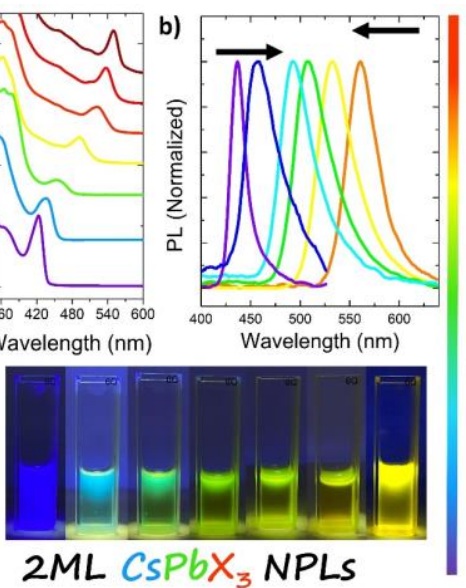

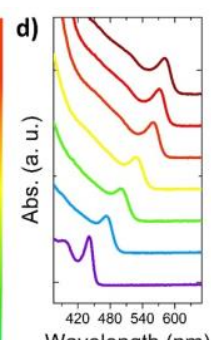

Wavelength $(\mathrm{nm})$

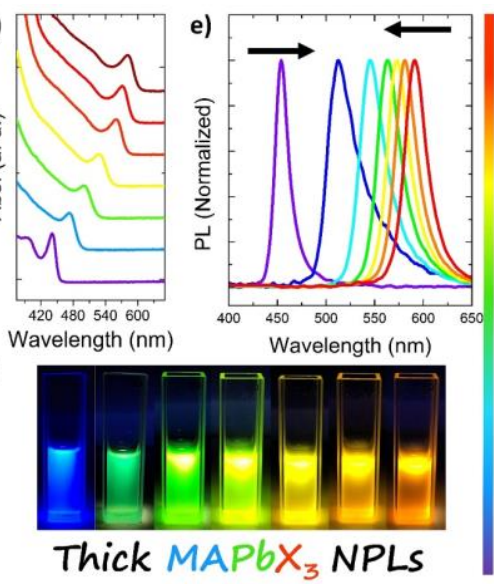

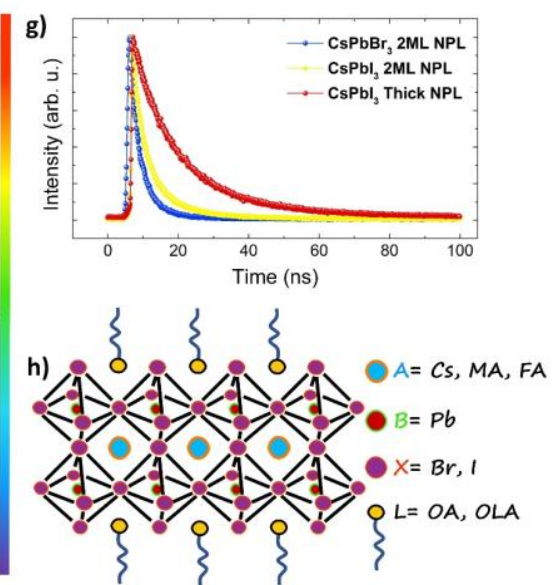

Figure 5. Tuning optical properties of colloidal metal halide perovskite nanoplatelets by changing the halide content in the perovskite nanoplatelets. Halide exchange reactions of

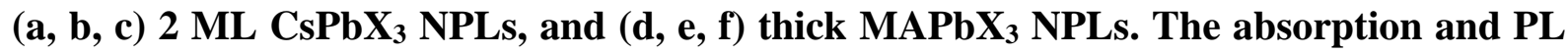
spectra of NPLs can be affectively tuned through variation of the halide composition of the nanoplatelets. The halide exchange reaction occurs by preserving thickness of the nanoplatelets. Figures (c) and (f) indicate the emission of 2 ML CsPbX 3 NPLs and thick $\mathrm{MAPbX}_{3}$ NPLs during the halide exchange reactions monitored under $350 \mathrm{~nm}$ UV light illumination. (g) Photoluminescence lifetime decay curves of $2 \mathrm{ML} \mathrm{CsPbBr}_{3}, \mathrm{CsPbI}_{3}$, and

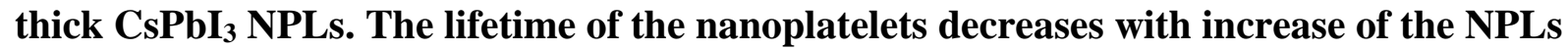
band gap. The band gap increment can be achieved by decreasing the thickness of the LHP NPLs or by changing the halide composition of LHP NPLs from I to Br or Br to Cl. For example, $\mathrm{CsPbBr}_{3}$ NPLs have a larger band gap than $\mathrm{CsPbI}_{3}$ NPLs for the same 
nanoplatelet thickness. (h) Schematic drawing of the crystal structure of the 2ML LHP NPLs. Each layer consists of $\mathrm{PbX}_{6}$ octahedrons. A-site cations allow formation of the perovskite unit cell. The ligands surrounding the nanoplatelets passivate the NPLs surface.

In order to tune optical properties of the colloidal nanoplatelets, we used the fast and facile anion exchange reaction approach in the perovskite nanoplatelets, see Figure 5. In the previous studies, postsynthetic anion exchange reactions were generally performed using chemicals such as octadecylammonium halides, oleylammonium halides, tetrabutylammonium halides, $\mathrm{PbX}_{2}$, and methylmagnesium halide salts. ${ }^{34,35}$ Different from the previous studies, here in this study, the synthesized seeds enable anion exchange reaction without preparation of any additional halide precursors. More importantly, the seed enabled halide exchange reaction provides fine tuning optical properties of LHP NPLs. In fact, the NLP thickness was preserved in the halide exchange reaction. In addition, by mixing the desired ratios of $\mathrm{PbI}_{2}$ and $\mathrm{L}_{2} \mathrm{PbBr}_{4}$ seed solutions in the seed-mediated synthesis, mixed halide NPLs were successfully synthesized. $2 \mathrm{ML} \mathrm{CsPbX}$ and thick $\mathrm{MAPbX}_{3}$ NPLs were used for anion exchange reactions, Figures 5a-5f. The mixed halide NPLs were prepared by adding $5 \mu \mathrm{L}$ of halide seeds to $2 \mathrm{~mL}$ of NPL solution. For example, in the halide exchange reaction of 2ML CsPbBr3 NPLs, we added $5 \mu \mathrm{L}$ of $\mathrm{PbI}_{2}$ seed solution for each step as shown in Figure 5. After the halide exchange reaction was completed in around 2 min., absorbance and PL spectra of the nanoplatelet colloid were acquired for each step. This process was repeated until the desired optical properties were obtained in the $2 \mathrm{ML} \mathrm{CsPbX} 3$ and thick $\mathrm{MAPbX}_{3}$ NPLs. Also, photoluminescence decay measurements, which are very useful tool for investigating the dynamics of the excitons in perovskite nanoplatelets, were performed for $2 \mathrm{ML} \mathrm{CsPbBr}_{3}, \mathrm{CsPbI}_{3}$, and thick $\mathrm{CsPbI}_{3} \mathrm{NPLs}_{\text {, }}$ Figure 5g. The PL lifetime of NPLs decreases with an increase in the band gap of NPLs, Figure 
5g. Note that the band gap of the NPLs can be engineered by controlling the thickness of the NPLs (quantum confinement effect) or by altering the halide composition of NPLs. For example, $\mathrm{CsPbBr}_{3}$ NPLs have a larger band gap than $\mathrm{CsPbI}_{3}$ NPLs for the same thickness value. The calculated average PL lifetimes from biexponential function fits are $18.81 \mathrm{~ns}, 9.17 \mathrm{~ns}$, and 5.05 ns for thick $\mathrm{CsPbI}_{3}, 2 \mathrm{ML} \mathrm{CsPbI}_{3}$, and $2 \mathrm{ML} \mathrm{CsPbBr}_{3} \mathrm{NPLs}$, respectively (see Table $\mathrm{S} 1$ for non-radiative and radiative recombination times and their corresponding percentages).

Table 1. Absorption, emission and PLQY properties of seed mediated synthesized APbX NPLs.

\begin{tabular}{|c|c|c|c|c|c|c|c|}
\hline & & \multicolumn{2}{|c|}{ Absorption } & \multicolumn{4}{|c|}{ Emission } \\
\hline & Thickness & $\mathbf{n m}$ & $\mathbf{e V}$ & $\mathbf{n m}$ & eV & $\begin{array}{c}\text { FWHM } \\
(\mathrm{meV})\end{array}$ & $\begin{array}{l}\text { QY } \\
(\%)\end{array}$ \\
\hline $\mathrm{L}_{2} \mathrm{PbBr}_{4}$ & $1 \mathrm{ML}$ & 397 & 3.12 & 405 & 3.06 & 201 & - \\
\hline $\mathrm{CsPbBr}_{3}$ & $2 \mathrm{ML}$ & 427 & 2.90 & 432 & 2.87 & 85 & 23.6 \\
\hline $\mathrm{CsPbBr}_{3}$ & Thick & 441 & 2.81 & 449 & 2.76 & 123 & 42.7 \\
\hline $\mathrm{MAPbBr}_{3}$ & $2 \mathrm{ML}$ & 428 & 2.89 & 432 & 2.87 & 90 & 32.6 \\
\hline $\mathrm{MAPbBr}_{3}$ & Thick & 445 & 2.78 & 451 & 2.75 & 97 & 60.8 \\
\hline $\mathrm{FAPbBr}_{3}$ & $2 \mathrm{ML}$ & 422 & 2.93 & 430 & 2.88 & 90 & 37.6 \\
\hline $\mathrm{CsPbI}_{3}$ & $2 \mathrm{ML}$ & 556 & 2.23 & 563 & 2.20 & 104 & 12.4 \\
\hline $\mathrm{CsPbI}_{3}$ & Thick & 577 & 2.14 & 592 & 2.09 & 106 & 33.8 \\
\hline $\mathrm{MAPbI}_{3}$ & $2 \mathrm{ML}$ & 566 & 2.19 & 572 & 2.17 & 90 & 4.14 \\
\hline $\mathrm{MAPbI}_{3}$ & Thick & 584 & 2.12 & 604 & 2.05 & 99 & 13.9 \\
\hline $\mathrm{FAPbI}_{3}$ & $2 \mathrm{ML}$ & 566 & 2.19 & 570 & 2.18 & 68 & 1.66 \\
\hline
\end{tabular}

In the final part, in Table 1, we summarize the absorption, PL, and QY properties of LHP NPLs synthesized using the seed mediated method developed in this study. The thinnest LHP NPL that can be synthesized by using seed mediated synthesis method is $1 \mathrm{ML} \mathrm{L}_{2} \mathrm{PbBr}_{4}$ NPLs. The $\mathrm{L}_{2} \mathrm{PbBr}_{4}$ NPL colloid has been used as a seed in this method. When $\mathrm{L}_{2} \mathrm{PbBr}_{4}$ crude solution was injected into hexane from the parent solution in $\mathrm{ODE}$, the $\mathrm{L}_{2} \mathrm{PbBr}_{4}$ aggregates to 
form larger particles within an hour. Stability of the particles can be greatly enhanced by increasing NPL thickness by A site cations. The $\mathrm{L}_{2} \mathrm{PbBr}_{4}$ NPLs synthesized in this study have $201 \mathrm{meV}$ emission linewidth, which is larger than the previously observed emission linewidths. ${ }^{8}$ The low stability and broader emission peak of $\mathrm{L}_{2} \mathrm{PbBr}_{4}$ NPLs may be caused by the lack of passivation on the this nanoplatelets. Absorption and emission spectra of the seed mediated synthesized NPLs have very similar and close peak positions to the previously reported absorption and emission peaks. ${ }^{8}$ However, a few differences have been observed. Firstly, almost all of the synthesized NLPs have slightly larger emission linewidths than previously synthesized

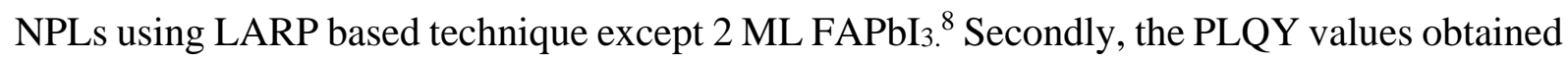
in this work are relatively higher than the previously obtained PLQY in 2ML NPLs. ${ }^{5,8,10,14}$ Thirdly, in $2 \mathrm{ML} \mathrm{APbBr} 3$ NPLs, cation size induced red shift was not observed. It should be noted here that larger cation in A site causes narrower bandgap in perovskite nanoplatelets ${ }^{8}$. Lastly, the 2 ML FAPbBr 3 NPLs have the largest band gap in the $2 \mathrm{ML} \mathrm{APbBr} 3$ NPLs and, the $\mathrm{FAPbBr}_{3}$ NPLs have $37.6 \%$ PLQY, which is the highest PLQY obtained in $2 \mathrm{ML} \mathrm{APbX}$ NPLs. It was observed that among the $2 \mathrm{ML} \mathrm{APbI}_{3} \mathrm{NPLs}$, synthesized in this study, $2 \mathrm{ML} \mathrm{CsPbI}_{3}$ had the highest PLQY, $12.4 \%$. On the other hand, thick $\mathrm{CsPbI}_{3}$ and $\mathrm{MAPbI}_{3} \mathrm{NPLs}_{\text {have }} 33.8 \%$ and $13.9 \%$ PLQY, and thick $\mathrm{CsPbBr}_{3}$ and $\mathrm{MAPbBr}_{3}$ NPLs have $42.7 \%$ and $60.8 \%$ PLQY, respectively. The highest PLQY obtained in this study is $60.8 \%$, which was observed in thick $\mathrm{MAPbBr} 3$ NPLs.

\section{CONCLUSIONS}

In summary, the seed mediated synthesis of $1 \mathrm{ML}, 2 \mathrm{ML}$, and thick colloidal metal halide perovskite NPLs was, for the first time, reported. The seed mediated synthesis of NPLs was conducted in hexane by reacting seed solution with A-site cation precursors (Cs-oleate, FAoleate, or diluted MA solution in hexane) at ambient conditions. The optical properties and 
morphologies of the seeds were thoroughly investigated. The high resolution transmission electron microscopy measurements reveal that the synthesized seeds are in a circular shape and have a size of around 2-3 nm diameter. In addition, the seed mediated growth of 2ML NPLs was studied by in situ optical absorption measurements, which indicate the optical properties of the colloid during the morphology of the colloid changes from NCs to NPLs. Upon transition from nanocrystal to nanoplatelet morphology, (i) sharpened excitonic absorption peaks, (ii) decrease in the linewidth of both absorption and emission peaks, and (iii) a red shift in the excitonic absorption peak were all observed. The ligand volume ratio (OLAM/OA) in the synthesis of seeds is very crucial for controlling the thickness and homogeneity of NPLs. 2ML $\mathrm{APbI}_{3}$ NPLs can only be synthesized using seeds with an OLAM/OA volume ratio of 2. Moreover, our facile and simple synthesis method provides highly stable, monodisperse NPLs with narrow absorption, photoluminescence line widths (68 meV and $201 \mathrm{meV}$ ), and high PLQY (37.6-1.66 \% for 2ML NPLs). In addition, by mixing presynthesized LHP NPLs with counter halide seeds, the optical properties of the NPLs can be affectively tuned by halide exchange reactions. We envision that the seed mediated synthesis of perovskite nanoplatelets reported here provides further understanding of the growth dynamics of LHP NPLs.

\section{METHODS}

Chemicals. Lead (II) iodide ( $\left.\mathrm{PbI}_{2}, 99 \%\right)$, Lead(II) Bromide $\left(\mathrm{PbBr}_{2}, \geq 98 \%\right)$ Cesium carbonate $\left(\mathrm{Cs}_{2} \mathrm{CO}_{3}, 99.9 \%\right)$, Formamidine acetate salt (FA-acetate, $\mathrm{HN}=\mathrm{CHNH} 2 \cdot \mathrm{CH} 3 \mathrm{COOH}$, 99,9\%), Methylamine solution (MA solution, 33 wt. \% in absolute ethanol), 1-octadecene (ODE, 90\%), oleylamine (OLAM, 70\%), octylamine (OcA, 99\%) oleic acid (OA, 90\%) and Hexane (EMPLURA®) were all purchased from Sigma-Aldrich and used without any further purification. 
Synthesis of Cs-Oleate. $350 \mathrm{mg}$ of $\mathrm{Cs}_{2} \mathrm{CO}_{3}, 20 \mathrm{~mL}$ of ODE, and $1.25 \mathrm{~mL}$ of OA were loaded in a round bottom glass flask and dried under vacuum for $1 \mathrm{~h}$ at $120^{\circ} \mathrm{C}$. After degassing, the temperature was increased to $150{ }^{\circ} \mathrm{C}$ under the flow of nitrogen gas and then Cs-oleate was obtained after $2 \mathrm{~h}$. Before its use in the synthesis of NPLs, Cs-oleate solution was heated to 100 ${ }^{\circ} \mathrm{C}$ in order to completely dissolve Cs-oleate in ODE.

Synthesis of FA-Oleate (method 1). $521 \mathrm{mg}$ of FA-acetate, $16 \mathrm{~mL}$ of ODE, and $4 \mathrm{~mL}$ of OA were mixed in a round bottom flask. The mixture was degassed at room temperature for $10 \mathrm{~min}$. and further degassed for $10 \mathrm{~min}$. at $80{ }^{\circ} \mathrm{C}$. Temperature of the reaction vessel was set to $135{ }^{\circ} \mathrm{C}$ under the nitrogen atmosphere and kept in that temperature until getting a clear solution. The FA-oleate solution was heated to $100{ }^{\circ} \mathrm{C}$ to completely dissolve FA-oleate in ODE.

Synthesis of FA-Oleate (method 2). $52 \mathrm{mg}$ of FA-acetate was dissolved in $2.5 \mathrm{~mL}$ of OA under ultrasonication and vacuum until a clear solution was obtained. The final solution was stored in the ambient condition for further use.

Synthesis of seeds. $2 \mathrm{~mL}$ of ODE, $0.2 \mathrm{mmol} \mathrm{PbX}_{2}\left(\mathrm{PbBr}_{2}\right.$ or $\left.\mathrm{PbI}_{2}\right)$ salt, $200 \mu \mathrm{L}$ of OLAM or $250 \mu \mathrm{L}$ of OcA, and $100-200 \mu \mathrm{L}$ of OA were loaded in a glass tube and degassed at $80{ }^{\circ} \mathrm{C}$ and subsequently, heated to $140-150{ }^{\circ} \mathrm{C}$ until all $\mathrm{PbX}_{2}$ solution became completely clear. Then, the colloid solution was immediately quenched to room temperature by immersing in an icewater bath.

$\boldsymbol{C s}_{\boldsymbol{B}} \boldsymbol{P} \mathrm{X}_{3}$ nanoplatelet synthesis. $\mathrm{CsPb}_{3} 2 \mathrm{ML}$ and thick NPLs were synthesized by mixing $\mathrm{PbX}_{2} \mathrm{NC}$ seed solution with Cs-oleate and then, adding this mixture into hexane. The desired amount of Cs-oleate was taken with a micropipette. Before adding Cs-oleate to $\mathrm{PbX}_{2}$ NC seed solution, Cs-oleate was cooled down to room temperature inside of the micropipette tip. When Cs-oleate became turbid white, it was added to the seed solution (This process is very 
crucial in order to prevent any early formation of large $\mathrm{CsPbX}_{3}$ nanocrystals!). Briefly, $100 \mu \mathrm{L}$ of $\mathrm{PbI}_{2} \mathrm{NC}$ seed solution was mixed with $5 \mu \mathrm{L}$ of Cs-oleate and then $60 \mu \mathrm{L}$ of this solution was injected into $5 \mathrm{~mL}$ hexane. This solution was stored at ambient conditions for 6 hours to complete the reaction. The same procedure was applied for the synthesis of thick $\mathrm{CsPbI}_{3} \mathrm{NPLs}$ except $20 \mu \mathrm{L}$ of Cs-oleate was used rather than $5 \mu \mathrm{L}$ of Cs-oleate. For quick synthesis of $\mathrm{CsPbI}_{3}$ 2ML NPLs, $50 \mu \mathrm{L}$ of seed solution and $2.5 \mu \mathrm{L}$ of Cs-oleate were injected respectively in 7.5 $\mathrm{mL}$ of hexane, followed by stirring at room temperature for 1 minute. The prepared solution was then placed in an oil bath at $45^{\circ} \mathrm{C}$ and magnetically stirred for 1.5 hours. Thick $\mathrm{CsPbI}_{3}$ NPLs can be synthesized using $7 \mu \mathrm{L}$ of Cs-oleate at the same temperature and time. $\mathrm{CsPbBr} 3$ 2ML NPLs were synthesized by mixing $100 \mu \mathrm{L}$ of $\mathrm{PbBr}_{2} \mathrm{NC}$ seed solution with $20 \mu \mathrm{L}$ of Csoleate and then $60 \mu \mathrm{L}$ of this solution was injected into $5 \mathrm{~mL}$ hexane, and finally the synthesized NPLs were stored overnight at ambient conditions before usage. For faster synthesis of $\mathrm{CsPbBr}_{3}$ 2ML NPLs, $50 \mu \mathrm{L}$ of OcA $(0.2 \mathrm{mmol} \mathrm{PbBr}, 250 \mu \mathrm{L}$ of OcA, and $200 \mu \mathrm{L}$ of OA in $2 \mathrm{~mL}$ ODE $)$ passivated seed solution and $7 \mu \mathrm{L}$ of Cs-oleate were injected respectively in $7.5 \mathrm{~mL}$ of hexane, followed by stirring at room temperature for 1 minute. The prepared solution was then placed in an oil bath at $65{ }^{\circ} \mathrm{C}$ and magnetically stirred for 2 hours. Thick $\mathrm{CsPbBr} 3$ NPLs can be synthesized using $15 \mu \mathrm{L}$ of Cs-oleate at the same temperature and time.

FAPbX 3 nanoplatelet synthesis (from FA-oleate method 1). FAPbX ${ }_{3} 2 \mathrm{ML}$ NPLs were synthesized by mixing $\mathrm{PbX}_{2} \mathrm{NC}$ seed solution with FA-oleate (method 1) and then, adding this mixture into hexane. The desired amount of FA-oleate was taken with a micropipette. Before adding FA-oleate to $\mathrm{PbX}_{2} \mathrm{NC}$ seed solution, FA-oleate (method 1) was cooled down to room temperature inside a micropipette tip (This process is crucial to prevent any early formation of large $\mathrm{FAPbX}_{3}$ nanocrystals!). Mainly, $100 \mu \mathrm{L}$ of $\mathrm{PbI}_{2} \mathrm{NC}$ seed solution was mixed with $6 \mu \mathrm{L}$ of FA-oleate (method 1) and then $60 \mu \mathrm{L}$ of this solution was injected into $5 \mathrm{~mL}$ hexane. The solution was inversion mixed and the reaction was complete in around 5 minutes. $2 \mathrm{ML}$ 
FAPbBr 3 NPLs were synthesized by mixing $100 \mu \mathrm{L}$ of $\mathrm{PbBr}_{2} \mathrm{NC}$ seed solution with $30 \mu \mathrm{L}$ of FA-oleate (method 1) and then $60 \mu \mathrm{L}$ of this solution was injected into $5 \mathrm{~mL}$ hexane. The solution was inversion mixed and kept for 4 hours at ambient conditions before usage.

FAPbBr $_{3}$ nanoplatelet synthesis (from FA-oleate method 2). $100 \mu \mathrm{L}$ of $\mathrm{L}_{2} \mathrm{PbBr}_{4}$ seed solution was added into $5 \mathrm{~mL}$ hexane. Subsequently, $15 \mu \mathrm{L}$ of FA-oleate (method 2 ) solution was injected into the solution and then the solution was immediately inversion mixed. The solution was kept at ambient conditions for 4 hours to complete the reaction.

$\mathrm{MAPbX}_{3}$ nanoplatelet synthesis. $0.5 \mathrm{~mL}$ of Methylamine solution (MA solution, $33 \mathrm{wt}$. $\%$ in absolute ethanol) was diluted with $9.5 \mathrm{~mL}$ of hexane for the synthesis $2 \mathrm{ML} \mathrm{MAPbX} 3$ NPLs. $100 \mu \mathrm{L}$ of $\mathrm{PbI}_{2} \mathrm{NC}$ solution was mixed with $7.5 \mu \mathrm{L}$ of diluted MA solution. Subsequently, $60 \mu \mathrm{L}$ of this mixture was injected into $5 \mathrm{~mL}$ hexane and then inversion mixed for the synthesis of $2 \mathrm{ML} \mathrm{MAPbI}_{3}$ NPLs. For the synthesis of $2 \mathrm{ML}$ MAPbBr3 NPLs, $100 \mu \mathrm{L}$ of $\mathrm{L}_{2} \mathrm{PbBr}_{4}$ seed solution and $70 \mu \mathrm{L}$ of diluted MA solution was mixed and $25 \mu \mathrm{L}$ of this mixture was injected into $5 \mathrm{~mL}$ hexane and then inversion mixed. It should be noted that the seeds were prepared by using OLAM did not support 2ML MAPbBr 3 NPLs and therefore, for the synthesis of $2 \mathrm{ML} \mathrm{MAPbBr} 3$, we used seeds passivated with $\mathrm{OcA}\left(0.2 \mathrm{mmol} \mathrm{PbBr}_{2}, 250 \mu \mathrm{L}\right.$ of $\mathrm{OcA}$, and $200 \mu \mathrm{L}$ of $\mathrm{OA}$ in $2 \mathrm{~mL}$ ODE). Thick $\mathrm{MAPbI}_{3}$ NPLs were synthesized using $1 \mathrm{~mL}$ of Methylamine solution (33 wt. \% in absolute ethanol) and $9 \mathrm{~mL}$ of hexane mixture as a MA source and $30 \mu \mathrm{L}$ of this solution was added to $100 \mu \mathrm{L}$ of $\mathrm{PbI}_{2}$ seed solution. $60 \mu \mathrm{L}$ of seed and MA mixture was injected into $5 \mathrm{~mL}$ hexane mixture and inversion mixed.

Characterization of Nanoplatelets. Scanning transmission electron microscopy (STEM) and transmission electron microscopy (TEM) analysis of NPLs were carried out in order to observe morphology of the nanocrystals (SEM; Tescan, GAIA 3, CZE), (TEM, JEOL2100F, Japan). The samples were prepared by drop-casting diluted NC suspensions onto 200 mesh carbon-coated copper grids. Absorption (Abs), Photoluminescence (PL), and time- 
resolved lifetime (LT) measurements were carried out by using a FS5 Spectrofluorometer (Edinburgh Instruments, UK). For PLQY, a Xenon lamp was employed with an excitation wavelength of $350 \mathrm{~nm}$. PLQY of each sample was determined by utilizing an integrating sphere. Samples were diluted in hexane and the optical properties of the nanocrystals were measured in a cylindrical quartz cuvette. In LT measurements, the samples were excited with a $350 \mathrm{~nm}$ laser with a pulse width of $100 \mathrm{ps}$ and a repetition rate of $1 \mathrm{MHz}$.

\section{CONFLICTS OF INTEREST}

There are no conflicts to declare.

\section{ACKNOWLEDGMENTS}

This research was supported by TUBITAK (118F523).

\section{REFERENCES}

1. Weidman, M. C.; Goodman, A. J.; Tisdale, W. A., Colloidal Halide Perovskite Nanoplatelets: An Exciting New Class of Semiconductor Nanomaterials. Chemistry of Materials 2017, 29, (12), 50195030.

2. Blancon, J.-C.; Even, J.; Stoumpos, C. C.; Kanatzidis, M. G.; Mohite, A. D., Semiconductor physics of organic-inorganic 2D halide perovskites. Nature Nanotechnology 2020, 15, (12), 969-985.

3. Guvenc, C. M.; Polat, N.; Balci, S., Strong plasmon-exciton coupling in colloidal halide perovskite nanocrystals near a metal film. Journal of Materials Chemistry C 2020, 8, (46), 1652016526.

4. Schmidt, L. C.; Pertegás, A.; González-Carrero, S.; Malinkiewicz, O.; Agouram, S.; Mínguez Espallargas, G.; Bolink, H. J.; Galian, R. E.; Pérez-Prieto, J., Nontemplate Synthesis of CH3NH3PbBr3 Perovskite Nanoparticles. Journal of the American Chemical Society 2014, 136, (3), 850-853.

5. Sichert, J. A.; Tong, Y.; Mutz, N.; Vollmer, M.; Fischer, S.; Milowska, K. Z.; García Cortadella, R.; Nickel, B.; Cardenas-Daw, C.; Stolarczyk, J. K.; Urban, A. S.; Feldmann, J., Quantum Size Effect in Organometal Halide Perovskite Nanoplatelets. Nano Letters 2015, 15, (10), 6521-6527.

6. $\quad$ Kang, C. H.; Dursun, I.; Liu, G.; Sinatra, L.; Sun, X.; Kong, M.; Pan, J.; Maity, P.; Ooi, E.-N.; Ng, T. K., High-speed colour-converting photodetector with all-inorganic $\mathrm{Cs} \mathrm{PbBr} 3$ perovskite nanocrystals for ultraviolet light communication. Light: Science \& Applications 2019, 8, (1), 1-12.

7. Zheng, X.; Hou, Y.; Sun, H.-T.; Mohammed, O. F.; Sargent, E. H.; Bakr, O. M., Reducing Defects in Halide Perovskite Nanocrystals for Light-Emitting Applications. The journal of physical chemistry letters 2019, 10, (10), 2629-2640.

8. Weidman, M. C.; Seitz, M.; Stranks, S. D.; Tisdale, W. A., Highly Tunable Colloidal Perovskite Nanoplatelets through Variable Cation, Metal, and Halide Composition. ACS Nano 2016, 10, (8), 7830-7839.

9. Huang, H.; Li, Y.; Tong, Y.; Yao, E. P.; Feil, M. W.; Richter, A. F.; Döblinger, M.; Rogach, A. L.; Feldmann, J.; Polavarapu, L., Spontaneous Crystallization of Perovskite Nanocrystals in Nonpolar 
Organic Solvents: A Versatile Approach for their Shape-Controlled Synthesis. Angewandte Chemie International Edition 2019, 58, (46), 16558-16562.

10. Bekenstein, Y.; Koscher, B. A.; Eaton, S. W.; Yang, P.; Alivisatos, A. P., Highly Luminescent Colloidal Nanoplates of Perovskite Cesium Lead Halide and Their Oriented Assemblies. Journal of the American Chemical Society 2015, 137, (51), 16008-16011.

11. Tyagi, P.; Arveson, S. M.; Tisdale, W. A., Colloidal organohalide perovskite nanoplatelets exhibiting quantum confinement. The journal of physical chemistry letters 2015, 6, (10), 1911-1916.

12. Bertolotti, F.; Nedelcu, G.; Vivani, A.; Cervellino, A.; Masciocchi, N.; Guagliardi, A.; Kovalenko, M. V., Crystal Structure, Morphology, and Surface Termination of Cyan-Emissive, Six-MonolayersThick CsPbBr3 Nanoplatelets from X-ray Total Scattering. ACS Nano 2019, 13, (12), 14294-14307. 13. Cho, J.; Choi, Y.-H.; O'Loughlin, T. E.; De Jesus, L.; Banerjee, S., Ligand-Mediated Modulation of Layer Thicknesses of Perovskite Methylammonium Lead Bromide Nanoplatelets. Chemistry of Materials 2016, 28, (19), 6909-6916.

14. Akkerman, Q. A.; Motti, S. G.; Srimath Kandada, A. R.; Mosconi, E.; D'Innocenzo, V.; Bertoni, G.; Marras, S.; Kamino, B. A.; Miranda, L.; De Angelis, F.; Petrozza, A.; Prato, M.; Manna, L., Solution Synthesis Approach to Colloidal Cesium Lead Halide Perovskite Nanoplatelets with Monolayer-Level Thickness Control. Journal of the American Chemical Society 2016, 138, (3), 1010-1016.

15. Sun, S.; Yuan, D.; Xu, Y.; Wang, A.; Deng, Z., Ligand-Mediated Synthesis of Shape-Controlled Cesium Lead Halide Perovskite Nanocrystals via Reprecipitation Process at Room Temperature. ACS Nano 2016, 10, (3), 3648-3657.

16. Wang, L.; Williams, N. E.; Malachosky, E. W.; Otto, J. P.; Hayes, D.; Wood, R. E.; GuyotSionnest, P.; Engel, G. S., Scalable Ligand-Mediated Transport Synthesis of Organic-Inorganic Hybrid Perovskite Nanocrystals with Resolved Electronic Structure and Ultrafast Dynamics. ACS Nano 2017, 11, (3), 2689-2696.

17. Vybornyi, O.; Yakunin, S.; Kovalenko, M. V., Polar-solvent-free colloidal synthesis of highly luminescent alkylammonium lead halide perovskite nanocrystals. Nanoscale 2016, 8, (12), 62786283.

18. Hassan, Y.; Song, Y.; Pensack, R. D.; Abdelrahman, A. I.; Kobayashi, Y.; Winnik, M. A.; Scholes, G. D., Structure-Tuned Lead Halide Perovskite Nanocrystals. Advanced Materials 2016, 28, (3), 566573.

19. Udayabhaskararao, T.; Kazes, M.; Houben, L.; Lin, H.; Oron, D., Nucleation, Growth, and Structural Transformations of Perovskite Nanocrystals. Chemistry of Materials 2017, 29, (3), 13021308.

20. Protesescu, L.; Yakunin, S.; Bodnarchuk, M. I.; Krieg, F.; Caputo, R.; Hendon, C. H.; Yang, R. X.; Walsh, A.; Kovalenko, M. V., Nanocrystals of Cesium Lead Halide Perovskites (CsPbX3, X $=\mathrm{Cl}, \mathrm{Br}$, and I): Novel Optoelectronic Materials Showing Bright Emission with Wide Color Gamut. Nano Letters 2015, 15, (6), 3692-3696.

21. Protesescu, L.; Yakunin, S.; Kumar, S.; Bär, J.; Bertolotti, F.; Masciocchi, N.; Guagliardi, A.; Grotevent, M.; Shorubalko, I.; Bodnarchuk, M. I.; Shih, C.-J.; Kovalenko, M. V., Dismantling the "Red Wall" of Colloidal Perovskites: Highly Luminescent Formamidinium and Formamidinium-Cesium Lead lodide Nanocrystals. ACS Nano 2017, 11, (3), 3119-3134.

22. Liu, L.; Huang, S.; Pan, L.; Shi, L. J.; Zou, B.; Deng, L.; Zhong, H., Colloidal Synthesis of CH3 NH3 $\mathrm{PbBr} 3$ Nanoplatelets with Polarized Emission through Self-Organization. Angew Chem Int Ed Engl 2017, 56, (7), 1780-1783.

23. Saliba, M.; Matsui, T.; Seo, J.-Y.; Domanski, K.; Correa-Baena, J.-P.; Nazeeruddin, M. K.; Zakeeruddin, S. M.; Tress, W.; Abate, A.; Hagfeldt, A., Cesium-containing triple cation perovskite solar cells: improved stability, reproducibility and high efficiency. Energy \& environmental science 2016, 9, (6), 1989-1997.

24. Chen, W.; Wu, Y.; Yue, Y.; Liu, J.; Zhang, W.; Yang, X.; Chen, H.; Bi, E.; Ashraful, I.; Grätzel, M., Efficient and stable large-area perovskite solar cells with inorganic charge extraction layers. Science 2015, 350, (6263), 944-948. 
25. An, M. N.; Park, S.; Brescia, R.; Lutfullin, M.; Sinatra, L.; Bakr, O. M.; De Trizio, L.; Manna, L., Low-Temperature Molten Salts Synthesis: $\mathrm{CsPbBr} 3$ Nanocrystals with High Photoluminescence Emission Buried in Mesoporous SiO2. ACS Energy Letters 2021, 6, 900-907.

26. Dutta, A.; Pradhan, N., Phase-stable red-emitting CsPbI3 nanocrystals: successes and challenges. ACS Energy Letters 2019, 4, (3), 709-719.

27. Guvenc, C. M.; Yalcinkaya, Y.; Ozen, S.; Sahin, H.; Demir, M. M., Gd3+-Doped $\alpha$-CsPbl3 Nanocrystals with Better Phase Stability and Optical Properties. The Journal of Physical Chemistry $C$ 2019, 123, (40), 24865-24872.

28. Zheng, Z.; Liu, A.; Wang, S.; Wang, Y.; Li, Z.; Lau, W. M.; Zhang, L., In situ growth of epitaxial lead iodide films composed of hexagonal single crystals. Journal of Materials Chemistry 2005, 15, (42), 4555-4559.

29. Pellegrino, G.; D’Angelo, S.; Deretzis, I.; Condorelli, G. G.; Smecca, E.; Malandrino, G.; La Magna, A.; Alberti, A., From PbI2 to MAPbI3 through Layered Intermediates. The Journal of Physical Chemistry C 2016, 120, (35), 19768-19777.

30. Papavassiliou, G. C.; Koutselas, I., Structural, optical and related properties of some natural three-and lower-dimensional semiconductor systems. Synthetic Metals 1995, 71, (1-3), 1713-1714.

31. Hang, P.; Xie, J.; Li, G.; Wang, Y.; Fang, D.; Yao, Y.; Xie, D.; Cui, C.; Yan, K.; Xu, J., An Interlayer with Strong Pb-Cl Bond Delivers Ultraviolet-Filter-Free, Efficient, and Photostable Perovskite Solar Cells. Iscience 2019, 21, 217-227.

32. Luo, Y.-R., Comprehensive handbook of chemical bond energies. CRC press: 2007.

33. De Roo, J.; Ibanez, M.; Geiregat, P.; Nedelcu, G.; Walravens, W.; Maes, J.; Martins, J. C.; Van Driessche, I.; Kovalenko, M. V.; Hens, Z., Highly Dynamic Ligand Binding and Light Absorption Coefficient of Cesium Lead Bromide Perovskite Nanocrystals. ACS Nano 2016, 10, (2), 2071-81.

34. Akkerman, Q. A.; D'Innocenzo, V.; Accornero, S.; Scarpellini, A.; Petrozza, A.; Prato, M.; Manna, L., Tuning the Optical Properties of Cesium Lead Halide Perovskite Nanocrystals by Anion Exchange Reactions. J Am Chem Soc 2015, 137, (32), 10276-81.

35. Nedelcu, G.; Protesescu, L.; Yakunin, S.; Bodnarchuk, M. I.; Grotevent, M. J.; Kovalenko, M. V., Fast Anion-Exchange in Highly Luminescent Nanocrystals of Cesium Lead Halide Perovskites (CsPbX3, $\mathrm{X}=\mathrm{Cl}, \mathrm{Br}, \mathrm{I}$ ). Nano Lett 2015, 15, (8), 5635-40.

\section{TOC FIGURE}

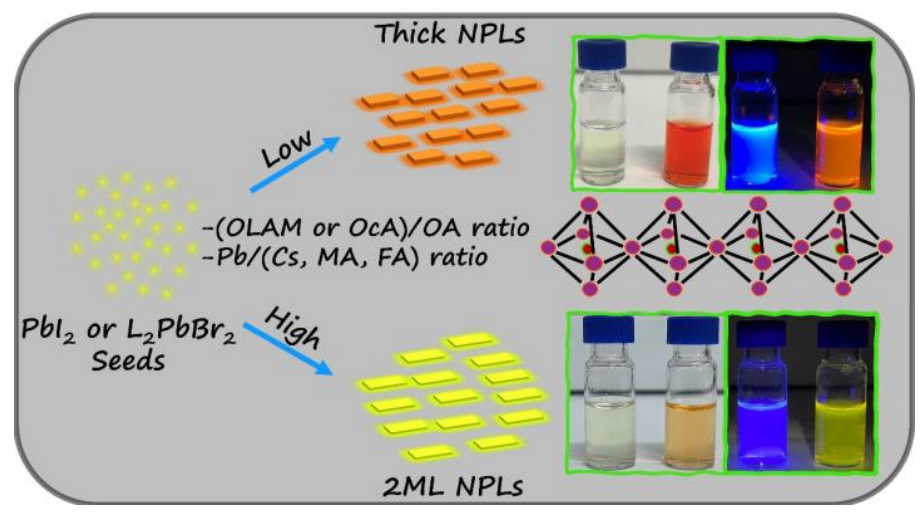

